\title{
Tourette syndrome and neuropsychological performance
}

Bornstein RA, Baker GB, Bazylewich T, Douglass AB. Tourette syndrome and neuropsychological performance.

Acta Psychiatr Scand 1991: 84: 212-216.

This study examined performance on a battery of neuropsychological tests in a sample of 28 patients with Tourette's syndrome (TS). Test scores were converted to age-corrected $T$-scores to control for the effect of age on test performance. The frequency of abnormal test performances was variable, but more frequent on motor and sensory tasks. Symptom severity as measured by the Tourette Syndrome Global Scale was inversely related to neuropsychological performance. In general, neuropsychological performance was mildly below average. The pattern of performance was similar to previous studies of TS patients.

\author{
R. A. Bornstein ${ }^{1,2}$, G. B. Baker ${ }^{2}$, \\ T. Bazylewich ${ }^{2}$, A. B. Douglass ${ }^{2,3}$ \\ ${ }^{1}$ Departments of Psychiatry, Neurology and \\ Neurosurgery, Ohio State University, Columbus, \\ Ohio, USA, ${ }^{2}$ Department of Psychiatry and \\ PMHAC Research Unit, University of Alberta, \\ Edmonton, Alberta, Canada, ${ }^{3}$ now at \\ Department of Psychiatry, University of \\ Michigan, Ann Arbor, Michigan, USA
}

Key words: Tourette syndrome; neuropsychological performance

R.A. Bornstein, Neuropsychology Laboratory, Ohio State University, 473 W. 12th Avenue, Columbus, $\mathrm{OH} 43210$, USA

Accepted for publication April 6, 1991
A number of studies (1-6) have reported evidence suggestive of mild cerebral dysfunction in patients with Tourette's syndrome (TS). These investigations have included reports of abnormalities in some patients on neurological examination, electroencephalogram, computerized axial tomography, magnetic resonance imaging and neuropsychological examination. Other studies have reported biochemical abnormalities in various bodily fluids including blood, urine and cerebrospinal fluid. In general, there has been consistent evidence of mild, but nonspecific brain abnormalities. There has been some consistency among the neuropsychological studies of patients with TS. Most studies (1-6) have reported abnormalities on tasks involving spatial, motor or graphic skills.

Most studies have reported IQs in the low average to average range (1-7), but patients with borderline normal $(6,8)$ as well as superior $(9)$ abilities have been reported. In addition to the level of intellectual ability, several studies have reported unusually high frequencies of large discrepancies between verbal IQ (VIQ) and performance IQ (PIQ). In the 4 studies that have provided data on the proportion of TS children demonstrating VIQ/PIQ discrepancies of 15 points or more, the proportion of such discrepancies has ranged from $25 \%$ to $55 \%(5-8)$. Discrepancies of this magnitude occur in approximately $10 \%$ of normal samples. The occurrence of a large VIQ/PIQ discrepancy is often interpreted in relation to other neuropsychological test findings as evidence of lateralized cerebral dysfunction. There has been some suggestion in the above studies for a higher incidence of PIQ greater than VIQ discrepancies. Discrepancies of this type are often found in patients with right hemisphere or bilateral dysfunction $(10,11)$.

In addition to studies of IQ patterns, some studies have included more detailed neuropsychological examinations. Three studies $(2,5,6)$ have reported the results of the Halstead-Reitan Battery. Incagnoli \& Kane (5) found that 11 of 13 subjects obtained overall performance scores within the normal range. That study also employed the Bender-Gestalt Test and found evidence of visual motor difficulties, which were also demonstrated in poor performance on the coding subtest of the Wechsler Intelligence Scale for Children-Revised (WISC-R). The pattern of diffculties on nonconstructional visuographic skills was interpreted to suggest disruption of cortical or basal ganglia function. Moldofsky \& Lazar (2) compared a group of $8 \mathrm{TS}$ children with groups of normal, learning disabled and acute brain-injured children. The TS patients were impaired on a number of sensory and motor tasks. On the Category Test, the TS children were not different from acute brain-damaged children, but were significantly worse than normals. The pattern of results suggested that, in spite of normal intelligence, the TS children in general demonstrated varying degrees of motor, perceptual, and conceptual abilities, although there were no localized or lateralized patterns of deficits. Bornstein et al. (6) 
also reported abnormalities on measures of motor, attention, and conceptual skills. The overall level of neuropsychological performance was within normal limits, although the pattern of abnormalities was interpreted to suggest a diffuse subcortical disturbance.

Most neuropsychological studies have been based on relatively small samples. In addition to small sample sizes, the studies of neuropsychological deficit in TS reported thus far have not in general considered level of neuropsychological performance, and none of the reports have attempted to relate ratings of TS symptoms with the neuropsychological abnormalities. This study was performed to address some of these concerns.

\section{Material and methods}

\section{Subjects}

The TS sample consisted of 28 ( 22 males, 6 females) subjects who met DSM-III-R criteria for TS, confirmed by board-certified child psychiatrists. The mean age of the subjects was 11.4 years $(\mathrm{SD}=3.9$, range 7-23) and the mean educational level was 4.9 years $(\mathrm{SD}=2.8)$. All patients were medication-free. Most patients had never been treated for their TS symptoms, and those that had been were medicationfree for a minimum of 2 weeks. Four of the TS patients were left-handed. Ratings of attention deficit disorder (ADD) and obsessive-compulsive disorder (OCD) were not obtained, although none of the TS patients met diagnostic criteria for these disorders.

\section{Neuropsychological measures}

The patients underwent a comprehensive psychological and neuropsychological test battery, which included the WISC-R, Peabody Picture Vocabulary Test (PPVT), Wide Range Achievement Test (WRAT), Knox Cube Test (KCT), Halstead Neuropsychological Test Battery for Children, TrailMaking Test (TMT), Wechsler Memory Scale, Grooved Pegboard Test and Reitan-Klove SensoryPerceptual Examination. Subjects over age $16(n=3)$ were administered the WAIS-R and adult forms of the Halstead-Reitan Battery.

The WISC-R, PPVT and WRAT yield scores that are corrected for age. The other measures yield raw scores that vary with age, and the version of the test also differs according to age. Because these raw scores vary considerably with age, analysis of raw data would be inappropriate. It was necessary to equate performance on these measures controlling for the effects of age. These raw scores were therefore converted to age-corrected $T$-scores (mean $=50$, $\mathrm{SD}=10)$ based on published normative data $(12,13)$, and computed so that scores above 50 re- flected above-average (for age) performance. In addition to correcting for the effects of age, the use of $T$-scores has additional advantages. Conversion of the data to a common metric permits comparison of performance between measures, and also permits computation of summary scores (such as mean $T$ scores). Such $T$-score averaging has previously been shown to be useful (14). Although a control group was not directly examined, conversion of the data to norm-based $T$-scores represents an implicit comparison with control values. Both normative studies used for data conversion were based on large samples of subjects recruited from the general population in 2 Canadian metropolitan areas. The mean scores on the neuropsychological tests in these 2 samples were very similar, which suggests that these scores represent reasonably accurate estimates of normative performance. The current sample also was drawn from a Canadian city, and it was considered that comparison of this sample with available norms represented a reasonable (if not ideal) method for evaluating neuropsychological performance of these patients with TS.

In addition to the neuropsychological measures, a rating of TS symptoms was obtained using the Tourette Syndrome Global Scale (TSGS) (15). The TSGS was performed by a trained research nurse who was aware of the neuropsychological test results and was conducted within 1 week of the neuropsychological examination. The TSGS provides measures of sample and complex motor tics as well as ratings of school and learning problems, restlessness and behavioral problems. A summary score is also computed. One psychometrist performed all of the neuropsychological examinations, and was unaware of the results of the TSGS.

It was of interest to examine the relationship between TS symptoms and neuropsychological performance. Because of large number of neuropsychological measures, it was necessary to reduce the number of correlations to be computed. The TSGS yields 7 scores, and there were 21 neuropsychological measures. It was decided to reduce the number of neuropsychological measures through the computation of average $T$-scores (14) that reflected performance across several tests. Several summary $T$-scores were computed that represented performance within particular domains of neuropsychological ability. These groups of variables were formed on the basis of similarities between the measures involved as well as previous factor analytic studies of the Halstead-Reitan Battery. A sensory score was computed that represented the average $T$-scores from both the right- and left-hand trials of finger agnosia and graphesthesia tests. The motor score was the average $T$-score from both hands trails of the Finger-Tapping Test and Grooved Pegboard 
Test. The cognitive score was the average $T$-score from the Halstead-Reitan measures including the Category Test, Tactual Performance Test-time, memory and location components, Seashore Rhythm Test, Speech Sounds Perception Test and TMT. Finally, a summary neuropsychological performance score was computed based on the average $T$-score across all measures.

\section{Results}

The mean scores for the neuropsychological measures are presented in Table 1. The mean IQ of the patients was in the average range (verbal IQ 95.8, performance IQ 96.3). It was first of interest to compare the patterns of neuropsychological performance with those reported in previous studies of TS patients. In this sample, the mean VIQ/PIQ discrepancy was $-0.54(\mathrm{SD}=14.5)$. Within the sample, 5 patients had VIQ $>$ PIQ discrepancies of 12 points or more, and another 5 had PIQ>VIQ discrepancies of 12 points or more. Consistent with previous studies (5-8), 36\% had large VIQ-PIQ discrepancies.

Most of the mean $T$-scores fall between the mean and one standard deviation below the mean. The only exceptions were the total time score from the Tactual Performance Test, right- and left-hand Finger Agnosia, and left-hand Graphesthesia. The latter was in large part caused by a single case with an

Table 1. Mean $T$-scores on neuropsychological variables for Tourette syndrome patients and percentage with abnormal scores

\begin{tabular}{lrrrrr}
\hline & Mean & \multicolumn{1}{c}{ SD } & $\leq 1$ SD & $\leq 1.5$ SD & $\leq 2$ SD \\
\hline Verbal IO & 95.8 & 9.4 & 11.5 & 7.7 & 3.8 \\
Performance IO & 96.3 & 13.7 & 19.2 & 11.5 & 0.0 \\
WRAT reading & 103.6 & 16.8 & 16.7 & 12.5 & 4.3 \\
WRAT spelling & 98.0 & 15.6 & 13.0 & 13.0 & 4.3 \\
WRAT arithmetic & 87.8 & 11.4 & 18.2 & 13.6 & 4.5 \\
Category $^{\text {TPT }}{ }^{3}$ time & 40.5 & 11.6 & 47.1 & 29.4 & 17.6 \\
TPT memory $_{\text {TPT location }}$ & 22.5 & 38.1 & 73.1 & 53.8 & 42.3 \\
Speech perception & 48.9 & 11.2 & 19.2 & 3.8 & 3.8 \\
Seashore rhythm & 45.1 & 8.8 & 23.1 & 15.4 & 3.8 \\
Trail making A & 52.6 & 8.6 & 10.5 & 5.3 & 0.0 \\
Trail making B & 50.3 & 10.1 & 9.5 & 9.5 & 4.8 \\
Finger tapping D & 43.3 & 25.7 & 19.2 & 7.7 & 7.7 \\
Finger tapping ND & 41.4 & 10.6 & 50.0 & 25.0 & 20.0 \\
Grooved pegboard D & 46.3 & 12.7 & 41.7 & 16.7 & 12.5 \\
Grooved pegboard ND & 44.6 & 20.3 & 19.2 & 15.4 & 11.2 \\
Graphesthesia D & 40.2 & 24.7 & 30.8 & 26.9 & 19.2 \\
Graphesthesia ND & 41.2 & 21.4 & 45.5 & 45.5 & 31.8 \\
Finger agnosia D & 36.2 & 38.9 & 36.4 & 27.3 & 22.7 \\
Finger agnosia ND & 22.8 & 56.7 & 60.9 & 56.5 & 34.8 \\
\hline
\end{tabular}

${ }^{1}$ Dominant hand.

${ }^{2}$ Non-dominant hand.

${ }^{3}$ Tactual performance Test. exceptionally poor score. In addition to examination of group means, it was also of interest to examine the performance of individual subjects. This was accomplished by examining the frequency of abnormal performance on each of the tests. Abnormal performance was defined in terms of deviations from normative means. Because different criteria for abnormality may be applied, Table 1 presents the proportion of subjects with $T$-scores of 40,35 and 30 $(-1 \mathrm{SD},-1.5 \mathrm{SD}$, and $-2 \mathrm{SD})$ on each variable.

There was considerable variability across tasks in the frequency of abnormal performances. Using the least stringent criteria ( $T$-score $\leqslant 40$ ), the proportion of abnormal scores ranged from $9 \%$ to $73 \%$. With the most stringent criteria ( $T$-score $<30$ ), the proportion of subjects with abnormal scores ranged from 0 to $42 \%$. When the criterion of 2 SD below normative means was used, the tests that had a high incidence $(10 \%)$ of abnormality were primarily motor and sensory-perceptual measures. The Halstead Category Test was the only exception.

Finally, it was of interest to examine the relationships between TS symptoms and neuropsychological performance (Table 2). There were consistent inverse relationships between TS symptom ratings and the neuropsychological measures. The global score from the TSGS was inversely related to the average $T$-score $(r=-0.44, P<0.05)$. In addition, the pattern of relationships appears to vary in regard to the nature of symptoms of neuropsychological measures. Table 2 indicates that simple phonic tics were associated with motor and sensory measures but not with more cognitive neuropsychological tasks. In contrast, complex motor symptoms were related to the more cognitive measures, and complex phonic tics appeared to be more consistently related to all aspects of neuropsychological performance (average $T$-score). As expected, school and learning problems were related to poor performance on the cognitive measures. The correlation between the average $T$-score and the restlessness rating from the TSGS approached significance $(P<0.10)$. Twentyfive percent of the correlations in this small sample

Table 2. Correlations between neuropsychological $T$-scores, summary scores and component scores from the TSGS neuropsychological $T$-scores

\begin{tabular}{lcccc}
\hline TSGS & Average T & Motor T & Sensory T & Cognitive T \\
\hline Simple motor & -0.30 & -0.25 & -0.24 & -0.22 \\
Complex motor & -0.23 & 0.13 & 0.04 & $-0.43^{1}$ \\
Simple phonic & -0.33 & $-0.39^{1}$ & $0.60^{2}$ & 0.14 \\
Complex phonic & $-0.46^{1}$ & -0.22 & $0.43^{1}$ & -0.31 \\
Behavior & 0.01 & -0.03 & 0.26 & -0.05 \\
Restlessness & -0.36 & -0.19 & -0.28 & -0.26 \\
Learning & $-0.43^{1}$ & 0.09 & -0.07 & $-0.54^{2}$ \\
\hline
\end{tabular}

${ }^{1} P<0.05 ;{ }^{2} P<0.01$. 
reach significance and there is a consistent pattern of inverse relationships between symptom severity and neuropsychological performance (Table 2). The number and consistency of the relationships is unlikely to be caused by chance alone.

\section{Discussion}

Consistent with previous studies of neuropsychological performance in patients with TS (1-6), this study provides evidence of mild abnormalities, primarily in motor and visuographic tasks. In addition, neuropsychological performance appeared to be related to TS symptoms, and the pattern of relationships seemed to vary with the nature of TS symptoms. Simple phonic tics were associated with motor and sensory tasks, but not with the more cognitive tasks. In contrast, the correlation between complex symptoms (motor and phonic) and the average neuropsychological $T$-score may suggest that these symptoms are associated with a broader range of neuropsychological performance. A similar pattern of relationships has been found in a large $(n=100)$ independent sample of TS patients (16).

The fact that the nature of neuropsychological abnormalities is consistent across studies lends some support to the notion that the deficits on these tasks reflect some process that is common to many patients with TS. In addition, the similarity of the patterns of relationships between neuropsychological measures and TS symptoms obtained in studies from different parts of North America (Alberta and Ohio) further supports the generalizability of these findings. However, there was considerable variability among the TS patients in the degree (as well as pattern) of performance, and most of the patients performed normally. Those abnormalities that were identified were relatively mild. Furthermore, the type of abnormalities observed are not specific to TS.

The finding of a relationship between neuropsychological performance and symptom ratings raises some question about the nature and meaning of this relationship. It is possible that both the primary TS symptoms and the neuropsychological deficit reflect the underlying pathophysiology of the disorder. On the other hand, the neuropsychological deficits may be a secondary effect. That is, the nature of the TS symptoms themselves may interfere with the performance of these tasks. This possibility seems unlikely because of the fluctuating nature of the symptoms, as well as the variability among patients with regard to the symptoms exhibited at any specific time. The fact that significant relationships in the anticipated direction were demonstrated lends support to the argument that the neuropsychological deficits are also a reflection of the underlying pathophysiology. The fact that the neuropsychological ab- normalities were demonstrated most clearly on motor and visuomotor tasks would appear to be consistent with previous studies (17) that have implicated basal ganglia structures in TS.

This sample was too small to permit direct examination of the effects of coexistent conditions such as OCD or ADD on neuropsychological performance. However, there is some reason to suggest that the current findings can not be attributed to these factors. None of the patients in the current sample met diagnostic criteria for either of these disorders. In addition, the restlessness score, which might be expected to reflect symptoms of ADD, was not significantly related to performance on the neuropsychological measures, although a trend in that direction was observed. This might be consistent with the reports $(7,9)$ of worse psychometric test performance in TS patients with ADD. With respect to OCD, there has been no published data indicating differences on neuropsychological measures in TS patients with or without OCD. A recent study (Bornstein, unpublished data) indicates that OCD characteristics do not account for the relationships observed in the current investigation. These data show that groups of TS patients with and without OC characteristics do not differ on the same neuropsychological measures used in this study. Furthermore, ratings of OCD symptoms in that study were not correlated with performance on neuropsychological measures. Therefore, it seems unlikely that OCD symptoms can account for the relationships in the current study.

In summary, these results suggest that, compared with available normative data, TS patients as a group performed slightly below average. The mean summary $T$-score (43.8) was less than one SD below the mean. Tests with relatively minor motor components had the best mean $T$-scores, whereas the TS patients were consistently poorer on motor and sensoryperceptual tasks. However, there was considerable variability in the level of performance among patients, and the summary neuropsychological performance score was inversely related to a rating of complex symptom severity. In addition, there was considerable variability between tests with regard to the percentage of TS patients with poor performances. Although the current investigation did not directly examine the effects of coexistent disorders on neuropsychological performance in TS, this should be examined in future studies. Finally, the contribution of TS-related factors, such as duration of illness, age at onset of symptoms, and previous or precipitating illnesses, should also be addressed. 


\section{Bornstein et al.}

\section{Acknowledgements}

The preparation of this manuscript was supported in part by grants from the National Institute of Mental Health (\# MH 42209) and the Alberta Provincial Mental Health Advisory Council (PMHAC). The technical assistance of Mrs. J. van Muyden, $\mathrm{S}$. Therrien, and Mrs. B. Murphy is gratefully acknowledged.

\section{References}

1. Thompson RJ, O’Quinn AN, Logue PE. Gilles de la Tourette's syndrome: a review and neuropsychological aspects of four cases. J Ped Psychol 1979: 4: 371-387.

2. Moldofsky H, Lazar L. Impaired neuropsychological functioning in Gilles de la Tourette's syndrome. Paper presented at International Neuropsychological Society, Mexico City, 1983. Unpublished.

3. Logue Pe, Platzek D, Hutzell R, Robinson B. Neurological, neuropsychological and behavioral aspects of Gilles de la Tourette's syndrome: a case. Percept Mot Skills 1973: 37: 855-861.

4. SAND, P. Neuropsychological test performance before and after symptom removal in a child with Gilles de la Tourette syndrome. J Clin Psychol 1972: 28: 596-600.

5. INCAGNOLI T, KANE R. Neuropsychological functioning in Gilles de la Tourette's syndrome. J Clin Neuropsychol 1981: 3: 165-169.

6. Bornstein RA, King G, Carroll A. Neuropsychological abnormalities in Gilles de la Tourette's syndrome. J Nerv Ment Dis 1983: 171: 497-502.
7. Shapiro AK, Shapiro ES, Young JG, Feinberg te. Gilles de la Tourette syndrome. New York: Raven Press, 1988.

8. Izmeth, A. Gilles de la Tourette's syndrome. J Ment Defic Res 1979: 23: 25-27.

9. Shapiro AK, Shapiro ES, Bruun RD, Sweet RD. Gilles de la Tourette's syndrome. New York: Raven Press, 1978.

10. Matarazzo JD. Wechsler's measurement and appraisal of adult intelligence: fifth and enlarged edition. New York: Oxford University Press, 1972.

11. Bornstein RA, Matarazzo JD. Wechsler VIQ versus PIQ differences in cerebral dysfunction: a literature review with emphasis on sex differences. J Clin Neuropsychol 1982: 4: 319-334.

12. KNIGHTS RM, Norwood JA. Revised smoothed normative data on neuropsychological test battery for children. Ottawa, Ontario: Carleton University, 1980

13. KLONOFF H, Low M. Disordered brain function in young children and early adolescents: neuropsychological and electroencephalographic correlates. In RETIAN RM, DAVISON LA, eds. Clinical neuropsychology: current status and applications. New York: John Wiley \& Sons, 1974: 121-178.

14. Kiernan RJ, Matthews CG. Impairment index versus $T$-score averaging in neuropsychological assessment. J Consult Clin Psychol 1976: 44: 951-957.

15. Harcherik DF, Leckman JF, Detlor J, Cohen DJ. A new instrument for clinical studies of Tourette's syndrome. J Am Acad Child Adolesc Psychiatry 1984: 23: 153-160.

16. BornsteIn RA. Neuropsychological performance in children with Tourette's syndrome. Psychiatry Res 1990: 33: 7381.

17. Chase TN, Geoffrey V, Gillespie M, Burrows GH. Structural and functional studies of Gilles de la Tourette syndrome. Rev Neurol 1986: 142 (11): 851-855. 